\title{
La responsabilité sociétale dans la construction d'indicateurs : l'expérience de l'industrie européenne de l'aluminium
}

\author{
Sylvie Faucheux, Isabelle Nicolaï ${ }^{\star}$ \\ Économistes, Université de Versailles Saint-Quentin-en-Yvelines, Centre d'économie et d'éthique pour l'environnement \\ et le développement (C3ED), UMR N 63 IRD-UVSQ, 47 Bd Vauban, 78047 Guyancourt cedex, France
}

L'importance politique et médiatique du développement durable conduit certaines entreprises à repenser, parfois à coûts supplémentaires, la question de leur responsabilité sociétale. Le présent texte, issu d'une recherche-action menée dans le cadre de l'Association européenne de l'aluminium, expose une méthode participative de construction de systèmes d'indicateurs et de suivi de la responsabilité sociétale des entreprises. Il est donc intéressant à double titre. Il constitue en premier lieu une fenêtre ouverte sur certaines activités initiées dans l'industrie à la faveur de l'affirmation du développement durable. Il est également intéressant car les méthodes utilisées privilégient la délibération entre les différentes parties prenantes, au sein des entreprises, mais aussi dans leur proche environnement. Il donne donc à voir une dynamique intéressante où des principes normatifs très généraux prennent corps dans un processus qui privilégie l'intervention des acteurs locaux.

La Rédaction

\footnotetext{
Mots-clés :

aluminium ;

concertation; développement durable ; environnement; focus groups; indicateurs; performance sociale ; responsabilité sociétale de l'entreprise
}

Keywords:
aluminium;
corporate social
responsibility;
deliberation;
environment;
focus groups;
indicators;
stakeholders;
social performance;
sustainable
development

\begin{abstract}
Social responsibility in the construction of indicators : the European steel industry experience. This paper presents methodological aspects and the main empirical results of a research study whose purpose was to define stakes and methods and propose appropriate indicators for defining and developing corporate social responsibility strategies for aluminium industry companies in Europe. Experimental empirical work for the testing of procedures for developing corporate social responsibility indicators was carried out at three industrial sites in France. At each of the three pilot sites, "focus groups" were constituted, one or two groups with "internal" stakeholders as employees, other groups with "external" stakeholders. The work of the groups generated indicator proposals, which were then classified in two ways. First, stakeholders' suggestions were gathered under categories established at international level covering both 'social' and 'environmental' dimensions of company responsibility. Second, the indicator suggestions were organised into a matrix with reference to (1) the category of stakeholder making the suggestion; (2) the site at which the suggestion was made; (3) the generic character versus specificity of the indicator. On the basis of this experience, recommendations for general application for the European aluminium industry are outlined.
\end{abstract}

^ Auteur correspondant : Isabelle.Nicolai@c3ed.uvsq.fr 
La responsabilité sociale des entreprises (RSE) se traduit par leur engagement à prendre en compte l'impact économique, social et environnemental de leurs activités pour les rendre compatibles avec les impératifs du développement durable. En affirmant leur responsabilité sociétale et en contractant de leur propre initiative des engagements allant au-delà des exigences réglementaires, les entreprises s'efforcent de répondre aux attentes émanant de toutes les parties prenantes ${ }^{1}$ : employés, investisseurs, consommateurs, fournisseurs, société civile, etc.

Les pressions se font de plus en plus fortes sur les entreprises afin qu'elles définissent une telle stratégie qui conduit également à des changements dans leur gestion interne comme l'ouverture à de nouvelles formes de dialogue et de consultation pour aider à la définition et à l'évaluation des décisions. Cette démarche s'inscrit dans ce qu'il est désormais courant de qualifier de gouvernance concertative ${ }^{2}$ et s'avère au cœur du Livre vert de la Commission européenne visant à « promouvoir un cadre européen pour la responsabilité sociale des entreprises » (18 juillet 2001). Ce Livre vert, qui sert désormais de référence, invite les entreprises, les pouvoirs publics à tous les niveaux, les organisations internationales, les partenaires sociaux et toute personne intéressée, à « exprimer leur opinion sur la manière de bâtir un partenariat destiné à ériger un nouveau cadre favorisant la responsabilité sociétale des entreprises, en tenant compte de leurs intérêts et de ceux de leurs diverses parties prenantes ».

Cet article, en se focalisant sur la définition et la mise en œuvre d'indicateurs de responsabilité sociétale pour l'industrie européenne de l'aluminium, s'inscrit dans cette problématique ${ }^{3}$. Les indicateurs de performances sociale et environnementale sont ici privilégiés, dans la mesure où les indicateurs de performances économiques existent depuis toujours dans les résultats annuels des entreprises ${ }^{4}$.

\footnotetext{
1 Parties prenantes ou stakeholders conformément à la définition proposée par Freeman (1984), Mitchell et al. (1997).

${ }^{2}$ Voir, par exemple, la convention d'Aarhus qui assure un droit à la participation des citoyens, la généralisation du dialogue social dans la régulation environnementale (éco-audit, accords volontaires...), ou encore la mise en œuvre des politiques de développement durable privilégiant la gouvernance concertative (principe 10 de la déclaration de Rio, agenda 21, $5^{\text {e }}$ et $6^{\text {e }}$ PCRD, Livre blanc de la Commission européenne sur la gouvernance du 25/07/01 ou le Livre vert sur la RSE $n^{\circ}$ COM 366 (2001)).

${ }^{3}$ Cette étude est issue de la recherche financée par l'AEA sur $l^{\prime} \ll$ Integration de la dimension sociale du développement durable au sein des entreprises de l'industrie d'aluminium : enjeux, méthodes, indicateurs et applications » (Faucheux et al., 2002a).

4 Ainsi la loi française sur les nouvelles régulations économiques, adoptée le 15 mai 2001, et son décret d'application du 21 février 2002, insiste bien sur la nécessité de définir des
}

La première section présente la méthode concertative de type bottom-up/top-down que nous avons développée pour construire de tels indicateurs. La seconde section offre les résultats empiriques obtenus grâce aux propositions faites par les différentes parties prenantes au cours de l'animation des focus groups réunis sur trois sites industriels sélectionnés afin de tester la méthodologie.

\section{Une démarche participative : la méthodologie bottom-up / top-down}

\section{Une justification empirique et conceptuelle de cette démarche}

Bien que la référence aux standards internationaux et sectoriels soit importante en vue d'une harmonisation et d'une cohérence des indicateurs de RSE, elle doit être complétée par une prise en compte des attentes et expériences des différentes parties prenantes. Cette prise en compte peut être assurée par le recours à une démarche concertative.

Un déficit de légitimité procédurale a souvent été invoqué par les critiques des approches décisionnelles traditionnelles de type top-down qui fonctionnent en vase clos entre décideurs et experts. La mise en place de procédures plus participatives de type bottom-up peut aider à pallier ce déficit (Encadré 1 ).

Il s'agit bien en pratique de s'intéresser non pas seulement à la légitimité de la décision, mais aussi à celle du processus décisionnel associé afin de déterminer des normes qui se définissent par rapport à un contexte so$\mathrm{cial}^{5}$. Une légitimation des choix sur des critères de gestion tels que des indicateurs nécessite la concertation des différents acteurs, sous la forme d'un arbitrage parfois conflictuel entre eux, afin d'identifier le champ des actions acceptables.

Cela signifie que les systèmes d'indicateurs et les procédures de reporting doivent être développés et appliqués en combinant des méthodes de participation bottom-up avec des processus de conception et de coordination topdown.

La méthodologie proposée, en accord avec l'industrie européenne de l'aluminium, autorise une interface des approches dites bottom-up reposant sur l'appréciation des préoccupations exprimées par les parties prenantes au cours de leurs parcours individuels, avec des analyses dites top-down faisant appel à des classifications

nouveaux indicateurs environnementaux et sociaux, notamment dans l'article 116.

5 «Selon l'éthique de la discussion, une norme ne peut prétendre à la validité que si toutes les personnes qui peuvent être concernées sont d'accord (ou pourraient l'être) en tant que participants à une discussion pratique sur la validité de cette norme. » (Habermas, 1986 [1983], p. 87.) 
Encadré 1. Pourquoi l'industrie européenne de l'aluminium recourt-elle à des procédures participatives dans le cadre de la définition d'indicateurs de RSE?

- Pour améliorer la qualité environnementale de la décision. La participation des différents stakeholders peut permettre de sortir du domaine restreint de l'expertise traditionnelle et d'intégrer au processus des compétences supplémentaires qui mettent en lumière d'éventuels effets pervers d'un choix technologique du point de vue environnemental (Funtowicz et Ravetz, 1993 ; Callon, 1998).

- Pour aboutir à des choix économiquement plus pertinents (tout en satisfaisant des critères environnementaux et sociaux). Les parties prenantes peuvent choisir les solutions qui offrent dans leur cas particulier un meilleur positionnement stratégique ou un meilleur rapport coût-efficacité, et éventuellement adapter ces solutions au gré de l'évolution de leur activité et des innovations technologiques (Haites et Yamin, 2000).

- Pour favoriser une approche transversale intégrant tous les champs politiques concernés dans une perspective de développement durable.

- Pour parvenir à une meilleure gestion des conflits. Ces conflits de pouvoir et/ou d'intérêts peuvent s'exprimer entre acteurs, entre les intérêts multiples d'un même acteur. Ils peuvent s'exercer sur différents problèmes d'environnement ou encore entre problèmes d'environnement et autres problèmes économiques et sociaux. Une approche participative permet aux différentes parties prenantes de confronter leurs représentations afin de faire émerger un accord à travers un affrontement constructif (O'Connor et Van den Hove, 2001).

- Pour modifier les perceptions du public et des parties prenantes sur les métiers de l'industrie de l'aluminium. La mise en place d'une démarche participative avec la totalité des acteurs offre plus d'information pour une meilleure éducation du public sur les problèmes environnementaux, de modifier les rapports de force et les conflits traditionnels, de changer les perceptions du contexte social, de renforcer l'implication des citoyens et de ce fait d'accroître la confiance des acteurs dans les institutions.

\begin{tabular}{|c|c|c|c|c|}
\hline TOP-DOWN & $\begin{array}{c}\text { Revue de la littérature } \\
\text { (publications, rapports } \\
\text { annuels de firmes, sites } \\
\text { internet, codes de } \\
\text { conduite et indices } \\
\text { boursiers...) }\end{array}$ & $\begin{array}{c}\text { Groupes de travail } \\
\text { composés des parties } \\
\text { prenantes dans le } \\
\text { secteur de l'aluminium }\end{array}$ & BOTTOM-UP \\
\hline $\begin{array}{c}\text { Systèmes d'indicateurs } \\
\text { élaborés à partir } \\
\text { d'hypothèses } \\
\text { académiques et des } \\
\text { standards } \\
\text { internationaux }\end{array}$ & & & & $\begin{array}{c}\text { Propositions } \\
\text { d'indicateurs } \\
\text { construites à partir des } \\
\text { perceptions locales des } \\
\text { problèmes }\end{array}$ \\
\hline
\end{tabular}

Fig. 1. La méthodologie bottom-up/top-down.

internationales en matière de responsabilité sociétale des entreprises (Fig. 1).

Pour valider un tel système de reporting bottom-up/topdown, nous avons mené conjointement :

- d'une part, une analyse systématique des stratégies de développement durable des entreprises et de mise en œuvre en leur sein de procédures participatives ;

- d'autre part, des travaux empiriques centrés sur trois études de cas sélectionnés en France en fonction des critères suivants : un emplacement territorial contrasté avec des contraintes et opportunités variées; une appartenance à des groupes industriels diversifiés présentant des cultures et des histoires particulières ; des activités variées dans le secteur de l'aluminium; une taille différente; des expériences diverses en matière de problèmes sociaux et/ou environnementaux.

Les trois sites retenus ont été les suivants :

- Pechiney à St-Jean-de-Maurienne (Savoie) spécialisé dans la production de fil machine, plaques et lingots ;

- Corepa SNC (une filiale de CFF Recycling) spécialisé dans le tri et la préparation des ferrailles d'aluminium à Bruyères sur Oise (Île-de-France) ;

- Building $\mathcal{E}$ Construction Systems (une filiale du groupe Alcoa Europe) à Merxheim (Alsace) spécialisé dans la fabrication de bandes ou tôles prélaquées, de panneaux multicouches d'aluminium. 
Encadré 2. Pourquoi l'industrie européenne de l'aluminium devrait-elle adopter le troisième modèle de responsabilité sociétale?

- La plupart des produits issus des entreprises du secteur de l'aluminium sont des produits intermédiaires et de ce fait peu familiers aux citoyens. Susciter des processus de dialogue avec toutes les « parties prenantes » concourrait à valoriser les actions des entreprises sur ce domaine et à leur donner une proximité avec le consommateur et la société civile. Cela aiderait aussi à mettre en évidence l'intérêt de l'aluminium dans les politiques de recyclage.

- Beaucoup d'entreprises de l'aluminium ont été confrontées dans le passé à de sérieux problèmes d'environnement et de santé. Elles ont beaucoup investi et souvent obtenu d'excellents résultats, mais sans que cela se sache. Ouvrir le dialogue permettrait ainsi à ces entreprises d'acquérir une réputation en conformité avec leur réelle pratique.

- Nombre d'entre elles ont connu des relations sociales tendues au cours de leur histoire, dues à des conditions de travail très difficiles. Là aussi beaucoup de progrès ont été accomplis sans que cela soit réellement discuté et donc connu.

- Certaines de ces entreprises ont eu des difficultés avec leurs activités dans les pays en développement, notamment au travers de leur exploitation de mines. Beaucoup travaillent en concertation désormais avec les populations des pays d'accueil pour mieux se conformer à leurs attentes et à leur mode de vie. Ce type de comportement n'est pourtant pas connu.

\section{Des modèles institutionnels de RSE différents}

L'acceptabilité sociale du résultat d'un processus décisionnel est intimement liée à la perception de la validité et de l'équité du processus, autrement dit à sa légitimité aux yeux des différents stakeholders. Cette méthode concertative permet aux parties prenantes, avec leurs préoccupations spécifiques par site, de voir comment leurs interrogations particulières et parfois locales peuvent être intégrées dans les catégories de responsabilité sociétale et les problèmes évoqués par la communauté internationale (De Marchi et al., 2000a ; WBCSD, 1999).

Au-delà de la possibilité qui s'ouvre à eux de rejeter des éléments non légitimes et peu équitables, les acteurs acquièrent, $\mathrm{du}$ fait de leur participation, une meilleure connaissance du processus. Cette connaissance, qui de facto induira une plus grande transparence, peut augmenter la crédibilité du processus aux yeux des différents acteurs et ainsi les motiver à mettre en œuvre les résultats de la décision sur le reporting (Armour, 1996). Toutefois, les modalités de participation des acteurs sont variables. Elles dépendent des modèles de responsabilité sociétale retenus.

Trois modèles contrastés ont ainsi pu être identifiés pour l'industrie européenne de l'aluminium :

- le premier considère que la RSE est assurée dans un contexte de négociation permanente entre salariés et direction organisée, si nécessaire, par l'État, afin de garantir de bonnes conditions d'emploi et d'avantages sociaux. Il s'agit d'une particularité française (mais plus largement sud-européenne) qui conditionne la construction des relations entre les parties prenantes. Dans notre étude empirique, il apparaît que ce modèle a été longtemps dominant sur le site de Pechiney et est encore très prégnant sur celui de Corepa SNC. Cependant, la situation évolue et de nombreuses parties prenantes pensent que ce modèle est de moins en moins souhaité et devrait s'estomper ;

- le second repose sur une substitution progressive de l'entreprise à l'État en matière de responsabilité sociétale. En accord avec les notions de libéralisme économique, la négociation et des accords directs se font entre les entreprises et la société civile au sujet des responsabilités sociétales. Par exemple, sur le site d'Alcoa, la pratique du mécénat d'entreprise est très forte et assure une gestion partielle des engagements de l'État par l'entreprise. Cette tendance est plutôt $\mathrm{d}$ 'inspiration anglo-saxone et ne reçoit pas nécessairement l'assentiment des parties prenantes des sites implantés en France ;

- le troisième modèle fait appel à une RSE réellement partagée et mise en place de manière concertée avec l'ensemble des parties prenantes (entreprises, État, société civile dans sa diversité, etc.). L'État intervient plus fortement que dans le modèle 2, mais en tant que partenaire et non plus comme arbitre (comme dans le modèle 1). Les formes de la responsabilité sociétale et leur traduction sont définies dans un partenariat social accepté par toutes les parties prenantes, que celles-ci soient internes ou externes. Les pays $\mathrm{d}^{\prime}$ Europe du Nord ${ }^{6}$ et, plus largement, la Commission européenne dans le Livre vert sur la responsabilité sociale des entreprises (COM 366 (2001)) tendent à promouvoir ce troisième modèle.

L'industrie européenne de l'aluminium devrait tendre vers ce troisième modèle. Susciter des processus de dialogue avec toutes les «parties prenantes » concourrait à valoriser les actions des entreprises (parfois déjà engagées) sur ces thèmes et à leur donner une proximité avec le consommateur ${ }^{7}$ et la société civile (Encadré 2).

\footnotetext{
${ }^{6}$ C'est cette conception que véhicule, par exemple, le groupe norvégien Norsk Hydro, producteur d'aluminium : «Notre rôle crée des obligations, mais dans le même temps nous devons délimiter et partager la responsabilité relativement aux autorités publiques et à la société civile et ne pas endosser leur propre responsabilité [... ]. » (Lunheim, 2001).

7 Par exemple, Maignan et Ferrell (2003) ont mis en avant les caractéristiques culturelles différentes des consommateurs européens vis-a-vis de la nature de la RSE.
} 
Tableau 1. Les principes de responsabilité sociétale exprimés par les parties prenantes.

\begin{tabular}{|c|c|c|c|c|c|c|c|}
\hline & \multicolumn{2}{|c|}{ Dimensions sociales } & \multicolumn{2}{c|}{$\begin{array}{c}\text { Dimensions } \\
\text { environnementales }\end{array}$} & \multicolumn{2}{c|}{$\begin{array}{c}\text { Type de concertation entre } \\
\text { parties prenantes }\end{array}$} \\
\hline & $\begin{array}{c}\text { La firme se } \\
\text { substituant à } \\
\text { l'État }\end{array}$ & $\begin{array}{c}\text { Interaction } \\
\text { entre } \\
\text { partenaires } \\
\text { sociaux }\end{array}$ & Sécurité/Santé & $\begin{array}{c}\text { Environnement } \\
\text { naturel }\end{array}$ & $\begin{array}{c}\text { Concertation } \\
\text { interne } \\
\text { Concertation } \\
\text { externe }\end{array}$ & $\begin{array}{c}\text { Concertation } \\
\text { multi-acteurs }\end{array}$ \\
\hline Pechiney & & $* * *$ & $* *$ & $* * *$ & $* * *$ & $* *$ & $* * *$ \\
\hline CFF & & $* * *$ & $*$ & $*$ & $* * *$ & $*$ & $* * *$ \\
\hline Alcoa & $* *$ & $* * *$ & $* * *$ & $* *$ & $* * *$ & $* *$ & $* * *$ \\
\hline
\end{tabular}

Légende : * faible intérêt; $\quad{ }^{* *}$ intérêt moyen; $\quad{ }^{* * *}$ fort intérêt manifesté dans les groupes. L'absence d'étoile signifie qu'aucune priorité n'a été donnée à la catégorie étudiée.

\section{Le choix du focus group comme méthode de concertation}

Dans notre recherche, la majorité des parties prenantes s'est exprimée en faveur d'un large processus de consultation pour l'établissement des objectifs, des stratégies et des indicateurs de développement durable. Le tableau 1 illustre l'intérêt porté par les parties prenantes pour les différentes dimensions du développement durable ainsi que la préférence des parties prenantes pour des stratégies faisant appel à une participation multiacteurs, traduite au travers d'un système de management transparent et participatif.

Différentes méthodes peuvent être utilisées pour animer les discussions, en particulier des supports documentaires (écrits ou images), des jeux de rôles, des techniques de libre association, des scénarios (réels ou prospectifs), ou encore des programmes informatiques interactifs (Faucheux et al., 2002a). Sur cette base, il s'agit de mettre en place des processus décisionnels qui tiennent compte des diversités d'intérêts, des inquiétudes légitimes du public et des considérations scientifiques, économiques ou éthiques et politiques. Les typologies de Beierle (2000) et Van den Hove (2001) montrent qu'il existe des degrés divers de participation des acteurs, des motivations très différentes pour celle-ci ainsi que des modes et formes de participation très variés. Une technique de plus en plus utilisée pour les questions de développement durable, de risques et d'environnement est celle des focus groups. Ce groupe de discussion comporte normalement 3 à 12 personnes avec un modérateur qui encourage les interactions entre participants et s'assure que la discussion reste centrée sur le sujet posé $^{8}$. L'objet de ce type de démarche est d'élucider les

\footnotetext{
${ }^{8}$ Il s'agit de techniques issues des sciences politiques et du marketing qui ont été développées et encouragées au sein des sciences sociales (Barbour et Kitzinger, 1999) par les entreprises,
}

attitudes et les priorités du public concernant une question et les décisions qui pourraient être prises et, sur cette base, de mettre en place des processus décisionnels qui tiendraient mieux compte des priorités et des inquiétudes légitimes des tous les acteurs (Stewart et Shamdasani, 1990).

Dans l'industrie de l'aluminium, des indicateurs environnementaux et sociaux existent déjà, et des systèmes de management respectant un certain nombre de procédures sont déjà généralisés. Les groupes de travail disposaient donc d'une certaine expérience. Le recours à la technique des focus group dans ces conditions s'est révélé fructueux pour les raisons suivantes :

- elle encourage, sur des questions connues, une participation d'acteurs afin que ceux-ci génèrent leurs propres questions, leurs cadres d'analyse et leurs concepts, et qu'ils poursuivent leurs propres priorités avec leurs propres termes, leur propre vocabulaire;

- le focus group permet dans certaines conditions d'augmenter le pouvoir d'influence ou de décision d'intérêts traditionnellement peu organisés ou d'acteurs dits faibles. Cette technique permet d'examiner les différentes perspectives des acteurs alors que ceux-ci opèrent dans un réseau social ;

- les procédures de mise en œuvre des focus groups ont été proposées par le modérateur et le mode d'interaction entre acteurs a été défini de façon endogène, par les participants eux-mêmes. De cette façon, le processus de sélection des indicateurs fonctionne de manière formelle afin de garantir les conditions de transparence et d'adoption de normes, tout en restant efficace car limitant les risques de contrôle par certains groupes;

les agences gouvernementales et les organisations internationales (O'Connoret et Meite, 2004). 
- le focus group accorde un intérêt tout particulier aux interactions entre acteurs à l'intérieur du groupe. Indépendamment de la façon dont ils sont sélectionnés, les participants ne sont jamais complètement homogènes. Le modérateur peut exploiter leurs différences d'opinion pour améliorer la qualité du processus de choix des indicateurs (Renn et al., 1993). Cela peut conduire à des solutions originales, mais peut également générer des malentendus et ouvrir la voie à un contrôle du processus par les acteurs les plus forts. Le modérateur doit alors veiller à ces principales limites.

\section{Des propositions d'indicateurs de RSE pour les firmes de l'industrie de l'aluminium}

\section{L'application de l'approche bottom-up à la sélection d'indicateurs}

Trois catégories de focus groups sur chacun des trois sites pilotes ont été constituées sur la base d'accords initiaux avec la direction des entreprises. Ces focus groups regroupaient un ensemble homogène de parties prenantes en fonction des attributs de pouvoir, d'urgence et de légitimité de chaque catégorie. Une distinction a été ainsi opérée entre trois principales catégories de parties prenantes:

- les parties prenantes internes (direction, employés, syndicats);

- les parties prenantes externes habituelles (fournisseurs, clients, actionnaires, banques, assurances) ;

- les parties prenantes externes élargies (ONG, collectivités territoriales, associations diverses, riverains, autres entreprises voisines, etc.).

À l'intérieur de chaque catégorie, plusieurs groupes de huit personnes en moyenne ont été sélectionnés sur la base du volontariat et du respect de l'anonymat dans la transcription des échanges, en accord avec le modérateur et l'entreprise. Sur les trois sites pilotes, la totalité du processus a ainsi rassemblé neuf focus groups au cours desquels plus de 60 personnes ont eu l'opportunité d'exprimer et d'échanger leurs vues et leurs suggestions d'indicateurs. Les profils des représentants des parties prenantes ont été variables selon les sites, en fonction des histoires sociales, des expériences de conflit liées à des problèmes environnementaux... Toutefois, les parties prenantes internes volontaires sont en général des acteurs investis par ailleurs dans la vie syndicale, associative à l'extérieur de l'entreprise, ou dans des responsabilités politiques. De plus, les parties prenantes externes les plus intéressées à une collaboration étaient déjà impliquées en partie dans les processus de décision. Ainsi, le processus de participation par focus group a permis de renforcer une dynamique déjà connue sur les sites.
La manière dont la décision de sélection d'indicateurs de développement durable est préparée et mise en œuvre importe autant que son contenu. Nous avons ainsi mené la procédure de pilotage selon quatre temps :

1. Le diagnostic et la pédagogie des enjeux : phase de sensibilisation. Tous les individus doivent avoir un accès identique à l'information pertinente concernant la question traitée, et une chance égale d'évaluer l'authenticité et la sincérité des déclarations et revendications des autres (Armour, op. cit.). Le questionnaire distribué comme support de discussion au focus group a été construit et présenté par le modérateur. La structure du questionnaire reflétait les buts poursuivis : rassembler les attentes des acteurs sur le reporting, obtenir une perception sur l'importance des différents acteurs et sur les différents aspects du cycle de vie de l'aluminium.

2. Le débat sur les différentes solutions : phase de participation et donc de responsabilisation ( $d$ 'une durée de 3 heures par groupe en moyenne). Pour obtenir des résultats, le modérateur doit être capable de surmonter la méfiance des participants et de clarifier leurs doutes. Les dialogues ont parfois amené les individus à de nouvelles réflexions afin de justifier ou de modifier une perspective. L'analyse institutionnelle a servi de cadre méthodologique pour comprendre la structure des intérêts de pouvoir et des conditions d'échange entre participants (De Marchi et al., 2000b).

3. Le choix arrêté par l'entreprise avec objectifs, calendrier et moyens : phase de contractualisation et d'engagement. Les propositions d'indicateurs ont été synthétisées, présentées et rediscutées par l'ensemble des parties prenantes impliquées dans les groupes de travail sur chacun des sites afin que les sélections finales soient véritablement validées.

4. La mise en œuvre participative et partagée : phase d'action exigeant l'évaluation et l'adaptation. Des propositions ont été formulées pour une mise en forme des indicateurs dans le rapport de développement durable du site, puis pour une agrégation des résultats au sein du groupe et plus largement du secteur européen. La direction conserve son autonomie sur la responsabilité du choix final. L'objectif est de réaliser un arbitrage entre la représentation des intérêts de toutes les parties prenantes pertinentes, et de réconcilier ces intérêts conflictuels. Cette phase est en cours de réalisation sur les différents sites pilotes.

Quant à la question souvent débattue de savoir si le modérateur doit être expert ou non, nous avons fait le choix d'intégrer un expert qui devait initier le débat au sein de focus group (nous plaçant de ce fait en contradiction avec la supposée neutralité avancée par les porteurs de l'analyse d'évaluation contingente (Mitchell et Carson, 1989)). Nous supposons que la présence d'un 
modérateur-expert améliore l'efficacité de la délibération du groupe et permet une réduction des coûts 9 .

\section{L'inventaire des indicateurs suggérés par les parties prenantes}

Une élaboration concertée des indicateurs par l'ensemble des parties prenantes a permis de sélectionner 85 indicateurs ( 48 sociaux et 37 environnementaux) et de renforcer leur qualité à partir des critères définis notamment par le Global Reporting Initiative (GRI, 2000, 2002), à savoir des critères de pertinence, fiabilité, clarté, comparabilité, opportunité, vérifiabilité. Les indicateurs sont référencés d'après un code : nous notons $\mathrm{S} 1, \mathrm{~S} 2, \mathrm{~S} 3 \ldots$ pour les indicateurs de dimension sociale et E1, E2, E3... pour ceux de la dimension environnementale.

Les listes de propositions d'indicateurs issues de nos études de cas ne prétendent pas à l'exhaustivité. Elles sont néanmoins suffisantes pour démontrer la richesse du potentiel disponible pour générer des indicateurs utiles pour les entreprises à travers des processus de participation des parties prenantes. Il est aisé de percevoir, à partir de ces listes révisées en concertation, la manière dont ces indicateurs peuvent être reliés aux classifications internationales standards (GRI). Par exemple, les parties prenantes ont sélectionné :

1. des indicateurs classiques de type GRI : ces choix portent surtout au niveau social sur les politiques de gestion interne des salaires et avantages. Pour de tels problèmes très codifiés et qui connaissent déjà souvent une clarification en interne, les parties prenantes ont retenu des indicateurs classiques du type "pourcentage de la masse salariale consacrée au comité d'entreprise» (S6), " existence d'une grille des salaires » (S7), «pourcentage de parts de capital détenues par les employés » (S8) ou encore sur les conditions de travail, le «taux de démission » (S14)... De même, au niveau des indicateurs environnementaux, la prise en compte des polluants et dommages associés à l'activité est très classique et a déjà fait l'objet d'une longue expérimentation dans les entreprises : «émissions de Fluor » (E4), "rejets d'eaux usées » (E13), «émissions de composés Nox » (E11), « coûts monétaires des dommages externes » (E18 qui est très proche de l'indicateur du GRI (2002) « conformité réglementaire » (EN16)) ou encore sur le système de

\footnotetext{
${ }^{9}$ Nous avons évalué le rôle de l'expert en répondant par l'affirmative aux deux questions posées par Aldred et Jacobs (2000) : le modérateur doit-il corriger les parties prenantes du groupe qui ont mal interprété une proposition donnée? Et si une des parties prenantes demande une information supplémentaire, le modérateur doit-il toujours la lui fournir, spécialement si cette transmission peut être affectée par le filtre personnel du modérateur?
}

management « existence d'une procédure de gestion de la qualité totale » (E31).

2. Des indicateurs plus inattendus : les parties prenantes ont retenu des indicateurs permettant de rendre compte des conditions de concertation à l'intérieur ou à l'extérieur de l'entreprise. Ainsi, l'indicateur (S25) permet d'obtenir «quel pourcentage d'employés ont été sollicités sur une question et quels ont été les résultats », l'indicateur (S28) retient la «fréquence des visites du site par le président du groupe », ou, sur le plan environnemental, les parties prenantes ont relevé l'indicateur « existence d'incitations et de récompenses pour les initiatives et innovations du personnel » (E33). De même, au niveau des relations avec les fournisseurs, les parties prenantes ont proposé «la proportion de contrats d'emploi de courte durée/temporaire par rapport à la main d'œuvre totale engagée " (S44) ou encore "la proportion de personnes qualifiées parmi les fournisseurs du site » (S46).

3. Des indicateurs centrés sur les préoccupations locales. Qu'il s'agisse des domaines social ou environnemental, le poids du local dans la définition des indicateurs est important dans la sélection menée. La méthode «bottom-up/top-down» contribue effectivement à faire émerger ce type d'indicateurs spécifiques. La réaction très positive et favorable des parties prenantes sur une telle sélection justifie l'utilisation d'une telle méthodologie. Par exemple, sur le plan social, on constate les indicateurs : "nombre d'employés originaires de la communauté du site» (S11), "taux d'emploi de jeunes locaux durant l'été» (S37), «fréquence des nouvelles concernant l'entreprise dans les lettres d'informations des autorités territoriales » (S39), ou encore « taxes locales payées par l'entreprise en proportion de l'ensemble des revenus de la ville ou de la région d'accueil » (S31); de même, pour les indicateurs de dimension environnementale, on note " mauvaises odeurs (signalées par des plaintes de la communauté locale) »(E10), « surface de terrain réhabilité comparée à la surface de terrain endommagé (référence historique locale) » (E28).

4. Des indicateurs qui exigent plus de précision et plus de transparence. Les parties prenantes ont souhaité sélectionner des indicateurs qui soient immédiatement interprétables, contrairement à certains indicateurs du GRI qui restent très flous quant à leur utilisation. Par exemple, le GRI (2002) retient comme indicateur-clé des relations avec la communauté une description seulement qualitative (SO1) qui évoque les «politiques mises en œuvre pour gérer les impacts de l'activité industrielle sur les zones voisines». Les parties prenantes dans nos études de cas ont préféré des indicateurs plus précis et concrets du 
type : "fréquence des visites du site par des classes d'école locale » (S40), « implication du directeur dans la communauté locale »(S41). De même, au niveau des conditions de travail et de santé-sécurité, les parties prenantes ont retenu le critère «pourcentage d'employés soumis à des examens de santé pour des maladies professionnelles graves » (S9), critère plus significatif que celui du GRI (2002) «pratiques sur l'enregistrement et la notification des accidents et maladies professionnelles ». Enfin, au niveau environnement, le GRI retient l'indicateur « utilisation de transport pour buts logistiques ». Les parties prenantes ont souhaité décomposer cet indicateur en mettant en évidence des choix stratégiques et technologiques bien spécifiques : elles ont retenu, dans cet esprit, les indicateurs : «nombre de camions arrivant/sortant par jour » (E8), « émissions de poussières » (E9), « bruit (nombre de plaintes reçues) » (E7) et « degré d'utilisation du transport par voie ferrée » (E32).

5. Des indicateurs parfois difficiles à évaluer. Afin de garantir l'utilisation de ces indicateurs, un protocole permettant de préciser les méthodes de mesures et calculs des principales catégories doit être retenu. Il est clair que, dans certains cas, les parties prenantes ont sous-estimé la difficulté de mettre en place de telles normes de calcul (comme par exemple, pour l'indicateur « existence d'un système de tri et/ou de recyclage des déchets » (E3)). Toutefois, ces indicateurs même difficilement calculables indiquent les préoccupations des parties prenantes, aussi les avons-nous laissés dans notre matrice de sélection présentée cidessous. À titre d'illustration, l'indicateur «pourcentage de messages circulant en français » (S30), très important sur le site de Merxheim, le critère « nombre de personnes reliées à un système de messagerie électronique » (S29) largement proposé sur le site de Pechiney, le "taux de rotation de l'équipe de direction » (S42) ou encore "l'existence d'objectifs environnementaux spécifiques différents de ceux établis par les autorités » (E23) sont difficilement interprétables mais représentent une information pertinente dans des contextes locaux et institutionnels très marqués.

6. Des indicateurs portant sur les mêmes objectifs d'entreprise mais avec des priorités ou perspectives différentes selon les groupes. Par exemple, sur l'axe gestion des déchets, l'objectif global est le même, mais les choix de trajectoire technologique sont différents (doit-on suivre le critère "modes de traitement et l'évolution des filières de recyclage associées » (critère (E3) retenu comme indicateur spécifique), ou doit-on s'en tenir à des objectifs de réduction des déchets générés par type de déchet ((E1) pour « le volume de déchets déposés » et (E2) pour « le volume de déchets traités et/ou récupérés » définis comme indicateurs génériques)?

\section{Une matrice pour la sélection des indicateurs}

Afin d'organiser les résultats des focus groups, une première classification des suggestions a été opérée en fonction des principaux systèmes internationaux d'indicateurs de développement durable pour les entreprises (GRI, Coalition for Environmentally Responsible Economies (CERES), World Business Council for Sustainable Development (WBCSD), SustAinability, World Resources Institute (WRI), etc.) en distinguant les dimensions sociales et environnementales. Ces indicateurs proposés par les parties prenantes sur tous les sites et proches d'indicateurs issus des classifications internationales sont dits "génériques ». Ils devraient a priori être pertinents pour l'industrie européenne de l'aluminium dans son ensemble.

Une seconde classification complémentaire à la première regroupe les indicateurs dans une matrice rendant explicite les origines contextuelles et l'échelle de pertinence des propositions (Tab. 2). À titre d'illustration, nous ne reprenons que les quelques indicateurs décrits ci-dessus.

Cette matrice permet l'identification des éléments suivants :

- la catégorie des parties prenantes à l'origine de la suggestion. En comparant ligne par ligne, il s'avère que les différentes catégories de parties prenantes sur un site donné fournissent des profils de suggestions largement similaires. Comme on pouvait s'y attendre, les stakeholders internes (en particulier les employés) se révèlent surtout préoccupés par les indicateurs relatifs aux conditions de travail ;

- le site sur lequel la suggestion a été émise ;

- le caractère spécifique de l'indicateur. Trois catégories de spécificité ont été introduites :

- la première concerne le type d'activité. Il apparaît très clairement des préoccupations concernant les aspects sociaux et environnementaux spécifiques à chaque type de production ou d'activité qui doivent être reflétées par des indicateurs sur mesure. Ces derniers sont a priori «transférables » pour toute entreprise européenne de l'aluminium relevant d'une activité similaire;

- la seconde catégorie de spécificité fait référence au profil socio-économique de l'usine dans sa région. Les deux sites de St-Jean et Merxheim constituent les principaux acteurs économiques locaux et sont assez éloignés des grandes villes. L'installation de Bruyère-sur-Oise, en revanche, est proche de Paris et se préoccupe moins de son insertion dans la communauté locale. Les indicateurs proposés par les groupes à St-Jean-de-Maurienne et Merxheim pourraient donc constituer des types pertinents généralisables à d'autres sites en Europe opérant 
Tableau 2. Quelques propositions d'indicateurs des parties prenantes — Une présentation synthétique.

\begin{tabular}{|c|c|c|c|c|c|c|c|c|c|c|c|c|c|c|c|c|c|c|c|c|}
\hline & \multirow{2}{*}{\multicolumn{2}{|c|}{$\begin{array}{l}\text { Indicateurs } \\
\text { génériques } \\
\text { pour le secteur } \\
\text { de l'aluminium }\end{array}$}} & \multicolumn{6}{|c|}{$\begin{array}{l}\text { Indicateurs spécifiques en fonction de } \\
\text { l'act ivité industrielle }\end{array}$} & \multicolumn{6}{|c|}{$\begin{array}{l}\text { Indicateurs spécifiques en fonction de } \\
\text { la situation géographique }\end{array}$} & \multicolumn{6}{|c|}{$\begin{array}{l}\text { Indicateurs spécifiques en fonction du } \\
\text { profil du groupe propr iétaire du site }\end{array}$} \\
\hline & & & \multicolumn{2}{|c|}{$\begin{array}{l}\text { Production / } \\
\text { Affinage } \\
\text { Transformation } \\
\text { primaire }\end{array}$} & \multicolumn{2}{|c|}{$\begin{array}{c}\text { Transformation } \\
\text { secondaire }\end{array}$} & \multicolumn{2}{|c|}{ Recyclage } & \multicolumn{2}{|c|}{$\begin{array}{l}\text { St-Jean-de } \\
\text { Maurienne }\end{array}$} & \multicolumn{2}{|c|}{ Merxheim } & \multicolumn{2}{|c|}{$\begin{array}{l}\text { Bruyères-sur- } \\
\text { Oise }\end{array}$} & \multicolumn{2}{|c|}{$\begin{array}{l}\text { St-Jean } \\
\text { Péchiney }\end{array}$} & \multicolumn{2}{|c|}{$\begin{array}{l}\text { Merxheim } \\
\text { Alcoa }\end{array}$} & \multicolumn{2}{|c|}{$\begin{array}{l}\text { Bruyères/Oise } \\
\text { CFF Recycling }\end{array}$} \\
\hline & Social & Envt & Social & Envt & Social & Envt & Soc. & Envt & Social & Envt & Social & Envt & Social & Envt & Social & Envt & Social & Envt & Social & Envt \\
\hline 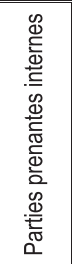 & $\begin{array}{l}\text { S25 } \\
\text { S39 }\end{array}$ & $\begin{array}{l}\text { E1 } \\
\text { E2 } \\
\text { E8 } \\
\text { E33 }\end{array}$ & S9 & $\begin{array}{c}\text { E3 } \\
\text { E4 } \\
\text { E9 } \\
\text { E11 } \\
\text { E13 } \\
\text { E32 }\end{array}$ & & $\begin{array}{l}\text { E3 } \\
\text { E10 } \\
\text { E13 }\end{array}$ & $\mathrm{S} 40$ & E9 & $\begin{array}{l}\text { S31 } \\
\text { S37 } \\
\text { S41 }\end{array}$ & E18 & $\begin{array}{l}\text { S31 } \\
\text { S37 } \\
\text { S41 }\end{array}$ & $\begin{array}{l}\text { E13 } \\
\text { E17 } \\
\text { E18 } \\
\text { E20 } \\
\text { E24 }\end{array}$ & & & $\begin{array}{l}\text { S6 } \\
\text { S11 } \\
\text { S29 } \\
\text { S42 }\end{array}$ & $\begin{array}{l}\text { E18 } \\
\text { E28 }\end{array}$ & $\begin{array}{l}\text { S7 } \\
\text { S8 } \\
\text { S14 } \\
\text { S29 } \\
\text { S30 }\end{array}$ & E31 & $\begin{array}{l}\text { S7 } \\
\text { S28 }\end{array}$ & E23 \\
\hline 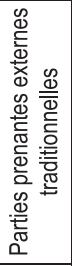 & S39 & $\begin{array}{l}\text { E1 } \\
\text { E2 } \\
\text { E8 } \\
\text { E33 }\end{array}$ & $\begin{array}{l}S 9 \\
S 44 \\
S 46\end{array}$ & $\begin{array}{c}\text { E3 } \\
\text { E4 } \\
\text { E9 } \\
\text { E13 } \\
\text { E32 }\end{array}$ & & $\begin{array}{l}\text { E3 } \\
\text { E10 } \\
\text { E13 }\end{array}$ & & & $\begin{array}{l}\text { S31 } \\
\text { S37 } \\
\text { S41 }\end{array}$ & E20 & $\begin{array}{l}\text { S31 } \\
\text { S37 } \\
\text { S41 }\end{array}$ & $\begin{array}{l}\text { E13 } \\
\text { E17 } \\
\text { E20 }\end{array}$ & & & $\begin{array}{l}S 6 \\
\text { S42 }\end{array}$ & $\begin{array}{l}\text { E18 } \\
\text { E28 }\end{array}$ & $\begin{array}{l}\text { S8 } \\
\text { S14 }\end{array}$ & E31 & & \\
\hline 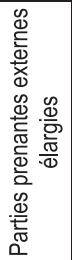 & $\begin{array}{l}\text { S25 } \\
\text { S39 }\end{array}$ & $\begin{array}{c}\text { E1 } \\
\text { E2 } \\
\text { E7 } \\
\text { E8 } \\
\text { E33 }\end{array}$ & s9 & $\begin{array}{c}\text { E3 } \\
\text { E4 } \\
\text { E9 } \\
\text { E11 } \\
\text { E13 } \\
\text { E32 }\end{array}$ & & $\begin{array}{l}\text { E3 } \\
\text { E10 }\end{array}$ & & & $\begin{array}{l}\text { S31 } \\
\text { S37 } \\
\text { S41 }\end{array}$ & E18 & $\begin{array}{l}\text { S31 } \\
\text { S37 } \\
\text { S41 }\end{array}$ & $\begin{array}{l}\mathrm{E} 17 \\
\mathrm{E} 20\end{array}$ & & & S42 & $\begin{array}{l}\text { E18 } \\
\text { E28 }\end{array}$ & S8 & & & \\
\hline
\end{tabular}

en milieu rural, avec des caractéristiques socioéconomiques comparables ${ }^{10}$;

- une dernière spécificité est attachée à l'identité du groupe industriel propriétaire ou contrôlant l'activité. Le groupe propriétaire et l'histoire de chaque site ont un impact évident sur la manière dont le développement durable est abordé. Ainsi, le site de St-Jean-de-Maurienne, détenu par le groupe français Pechiney ${ }^{11}$, est marqué par une longue histoire et une culture d'entreprise ancrées dans le «paternalisme » communautaire de l'entreprise du $19^{\text {e }}$ siècle et dans la «lutte des classes », opposant direction et employés, typique du milieu du

\footnotetext{
10 Peu d'indicateurs environnementaux démontrent des spécificités géographiques élevées. Par exemple, à Merxheim, les indicateurs types « rejets de PCB » (E17) et "coûts monétaires des dommages externes » (E18) sont proposés pour illustrer la priorité accordée au maintien de la qualité des ressources aquifères en Alsace, et les types «quantité d'eau utilisée par unité de production » (E20) et «proportion d'employés dans le service environnement » (E24) reflètent les conditions géographiques spécifiques à la qualité de l'air.

11 À ce titre, il sera intéressant d'analyser les conséquences du rachat de Pechiney annoncé en 2003 par le groupe canadien Alcan.
}

$20^{\mathrm{e}}$ siècle. Certains des indicateurs proposés sur ce site sont caractéristiques de cet héritage. Le site de Merxheim, quant à lui, n'a que très récemment été racheté par le groupe américain Alcoa (en 2000). Auparavant, il était détenu et géré par le groupe Reynolds, également américain, mais dont le style de gestion s'avérait plus décentralisé. Les activités syndicales y sont peu développées. Cet aspect se reflète dans la manière dont les principes de responsabilité sont exprimés. Enfin, le site de CFF (Corepa SNC) à Bruyères-sur-Oise a toujours été géré comme une PME alors que ses activités se sont considérablement développées au cours de la dernière décennie, ce qui rend les liens de communication et de dialogue assez tendus.

\section{Une interprétation des résultats}

Une telle approche bottom-up/top-down permet d'évaluer jusqu'à quel point chaque indicateur a une signification locale spécifique ou peut être utilement généralisé à une autre échelle (groupe, secteur. . .).

Par exemple, au niveau environnemental, nous avons constaté des préoccupations hétérogènes et des 
spécificités de sites très intéressantes à mettre en valeur pour une interprétation à une échelle plus large. Par exemple :

- les parties prenantes liées au site de Pechiney sont apparues davantage intéressées par la qualité de l'environnement naturel que par les problèmes de santé et de sécurité. Plusieurs facteurs peuvent expliquer ce constat : le type d'activité sur le site de St-Jeande-Maurienne; la disparition des maladies professionnelles depuis environ 20 ans, la longue histoire du site pour laquelle la mémoire collective a encore clairement à l'esprit les impacts préjudiciables d'une époque où les investissements anti-pollution n'existaient pas encore; la géographie du site (replié dans une vallée) ; et, enfin la place importante déjà accordée aux mesures de santé et de sécurité dans les accords généraux sur l'emploi et les conditions de travail sur le site ;

- les parties prenantes internes sur le site de CFF à Bruyères-sur-Oise accordent peu d'importance aux problèmes environnementaux en général. Elles sont relativement plus focalisées sur la sécurité et les questions de santé des employés. Ce profil de préoccupations s'explique, de nouveau, en fonction des spécificités du site : le type d'activité (recyclage des carrosseries d'automobiles); les problèmes de gestion liés au fonctionnement d'une activité importante avec les moyens d'une petite entreprise ; la proximité de Paris et une communication interne hésitante ;

- les parties prenantes du site d'Alcoa se révèlent très sensibilisées aux aspects de santé et de sécurité. Les facteurs pouvant expliquer cette attitude sont les suivants : une production secondaire d'aluminium bien moins polluante par unité d'activité ; l'âge relativement jeune de l'installation (établie dans les années 1960); la gestion de type américain insistant sur la « qualité-sécurité » mise en œuvre par les propriétaires successifs du site (Reynolds puis Alcoa); et enfin, une longue tradition de dialogue avec la communauté externe.

De même, les indicateurs proposés pour les dimensions relatives à la dimension sociale témoignent de profils institutionnels spécifiques, non seulement de chaque culture nationale, mais également de chaque site. À titre d'illustration, les groupes du secteur de l'aluminium de gestion anglo-saxonne ont tendance à privilégier, parmi les indicateurs sociaux, ceux relatifs à la parité sexuelle ou ethnique. Or, nous avons pu constater que pour les entreprises de ces groupes opérant en France, ce type d'indicateurs est mal perçu par les parties prenantes car ils reviennent à mettre en place des politiques de « discrimination positive », ce qui est contraire aux systèmes du droit du travail français.
En outre, cette méthodologie permet de réduire considérablement le nombre $\mathrm{d}^{\prime}$ indicateurs à retenir ${ }^{12}$. Le système de reporting devient alors beaucoup plus efficace car plus maniable et plus adapté au contexte. Les parties prenantes du secteur de l'aluminium ont proposé de limiter l'ensemble des indicateurs (sociaux et environnementaux) à une trentaine par site afin de conserver sa transparence et sa pertinence. Bien qu'elles plaident en faveur d'une vision intégrée de la responsabilité sociétale de l'entreprise, elles ne recherchent pas pour autant des indicateurs composites. Peu d'acteurs sont convaincus de la vertu des indicateurs « intégrés » prétendant capturer ensemble dans un seul indice toutes les dimensions des performances sociales et/ou environnementales. Elles avancent davantage la nécessité de travailler avec un éventail bien équilibré d'indicateurs, chacun étant clairement approprié à l'aspect spécifique de la responsabilité sociétale traité.

Enfin, cette méthodologie garantit le caractère opérationnel du système de reporting, dans la mesure où les indicateurs choisis ont fait l'objet d'une validation par l'ensemble des parties prenantes. Compte tenu des caractéristiques retenues dans la matrice d'analyse, nous relevons que des indicateurs spécifiques à un site du secteur de l'aluminium peuvent :

- compléter des indicateurs génériques pour l'industrie de l'aluminium dans son ensemble. Par exemple, les indicateurs proposés à Merxheim pourraient être appliqués sur des sites établis depuis longtemps dans des petites villes ou en milieu rural, que ce soit dans le Nord ou le Sud de l'Europe ;

- constituer des liens permettant aux employés et aux parties prenantes locales de percevoir la pertinence du système d'indicateurs retenus et donc d'adhérer à un système de reporting qui les synthétiserait.

\section{Conclusion}

Une entreprise du secteur de l'aluminium qui se contenterait d'appliquer des indicateurs décidés au niveau du siège social ou proposés par des agences internationales rencontrerait des obstacles provenant, tant d'un manque de compréhension de la part des parties prenantes locales sur ce que les indicateurs sont supposés mesurer et sur la manière dont ils correspondent à leurs intérêts, que de l'inadéquation des procédures standardisées par rapport à la situation donnée.

La méthodologie développée dans le cadre de cet article constitue un outil de gestion multicritère permettant

\footnotetext{
12 Le GRI (2002) retient 97 indicateurs qui restent parfois très qualitatifs et donc difficilement utilisables par les parties prenante, alors que notre double système de classification permet de n'en retenir qu'une cinquantaine définie pour le groupe sur chaque site.
} 
de comparer différents scénarios construits en fonction des objectifs de développement durable établis par les entreprises ou de leurs conséquences possibles.

Toutefois, cette pratique concertative peut présenter quelques limites :

- l'apprentissage du dialogue avec les parties prenantes est un processus qui peut s'avérer long et complexe en fonction de la nature des personnels, de l'industrie, du contexte social, de la nature des problèmes environnementaux ou du contexte économique ;

- une des limites de cette méthode est la représentativité limitée de l'échantillon dans un problème de choix collectif. Toutefois, ce qui nous intéresse dans cette démarche, ce n'est pas la représentativité statistique, mais la tentative d'anticiper les perspectives particulières privées qui pourraient influencer les preneurs de décision et s'assurer que toutes les perspectives ont été intégrées de manière adéquate;

- le poids des indicateurs sociaux peut paraître important par rapport aux indicateurs environnementaux. Nous pensons que le contexte social très particulier de l'industrie de l'aluminium et les efforts déjà exercés sur les aspects environnementaux peuvent justifier cette caractéristique ;

- la mise en place et le suivi concret des indicateurs est un processus long et difficile. Pour l'instant, l'industrie de l'aluminium nous a confié une seconde phase de travail consistant à appliquer la méthodologie bottom-up/top-down à d'autres sites européens afin de perfectionner la distinction générique/spécifique sur la base d'expériences comparatives à travers plusieurs pays européens (Grande-Bretagne, Italie, Allemagne), et d'expérimenter pour les sites sélectionnés la mise en œuvre de tels systèmes d'indicateurs et de procédures de reporting adaptés aux différents niveaux du site, du groupe et du secteur européen de l'aluminium dans son ensemble (Faucheux et O'Connor, 2003);

- si les focus groups peuvent améliorer la base d'information pour les décideurs, il est plus rare cependant qu'ils jouent un rôle direct de formulation ou d'évaluation d'une décision. Le suivi du système de reporting devient dès lors un enjeu pour le succès d'une telle démarche.

\section{Remerciements}

Nous remercions l'Association européenne de l'aluminium qui a financé la recherche "Integration de la dimension sociale du développement durable au sein des entreprises de l'industrie d'aluminium : enjeux, méthodes, indicateurs et applications » dont les résultats sont rapportés ici. Nous remercions nos différents interlocuteurs, les directions des entreprises, les employés et les membres des communautés locales grâce à qui nous avons pu mener à bien nos focus group. Nous exprimons aussi notre gratitude aux membres du comité de pilotage de ce travail issus de l'AEA, de Pechiney, de la Fédération française des métaux non ferreux et du cabinet Communication et Institutions.

\section{Références}

Aldred, J., Jacobs, M., 2000. Citizens and Wetlands: Evaluating the Ely Citizens' Jury, Ecological Economics, 34, 217-232

Armour, A., 1996. The Citizens' Jury Model of Public Participation, in Renn, O., Webler, T., Wiedemann, P. (Eds), Fairness and competence in Citizen Participation: Evaluating Models for Environmental Discourse, Dordrecht, Kluwer.

Barbour, R., Kitzinger, J. (Eds), 1999. Developing Focus Group Research, Newbury Park, Sage Publications.

Beierle, T.C., 2000. La qualité des décisions impliquant des stakeholders : leçons à partir de cas d'études, Resources for the Future, Washington, D.C. "Discussion Paper", 00-56.

Callon, M., 1998. Des différentes formes de démocratie technique, Annales des Mines, 9, 63-73.

Commission européenne, 2001. Livre vert «Promouvoir un cadre européen pour la responsabilité sociale des entreprises », COM (2001) 366 final, juillet.

Faucheux, S., Hue, C., Nicolaï, I., O'Connor, M., 2002a. Integration of the Social Dimension of Sustainable Development in Enterprise Strategies within the Aluminium Industry, C3ED final report, EAA Programme "Aluminium for Future Generations", Guyancourt, France.

Faucheux, S., Hue, Ch., Petit, O., 2002b. NTIC et Environnement, enjeux, risques et opportunités, Futuribles, 273, 3-26.

Faucheux, S., O'Connor, M., Spangenberg, J., de Marchi, B., 2003. Implementation of a System of Indicators for Social Responsibility Reporting, C3ED final report, EAA Programme "Aluminium for Future Generations", Guyancourt, France.

Freeman, R.E., 1984. Strategic Management: a Stakeholder Approach, Boston, Pitman/Ballinger.

Funtowicz, S., Ravetz, J., 1993. Science for the Post-Normal Age, Futures, 25, 7, 735-755.

Global Reporting Initiative, 2000. Sustainability Reporting Guidelines on economic, environmental and social performance, June.

Global Reporting Initiative, 2002. Sustainability Reporting Guidelines, http://www.globalreporting.org/ GRIGuidelines/2002/gri_2002_guidelines.pdf.

Habermas, J., 1986 [1983]. Morale et communication, Paris, Les Éditions du Cerf, trad. de Moralbewusstsein und Kommunikatives Handeln, Frankfurt am Main, Suhrkamp Verlag.

Haites F., Yamin F., 2000. The Clean Development Mechanism: proposal for its operation and governance, Global Environmental Change, Part A, 10, 1, 27-45.

Lunheim, R., 2001. Taking social responsibility to the world, Profile, 2, Norsk Hydro, May.

Maignan, I., Ferrell, O.C., 2003. Nature of corporate responsibilities; Perspectives from American, French, and German consumers, Journal of Business Research, 56, 55-67. 
Marchi, B. de, Funtowicz, S., Guimaraes Pereira, A., 2000a. From the Right to Be Informed to the Right to Participate: Responding to the Evolution of the European Legislation with ICT, International Journal of Environment and Pollution, $15,1,1-21$.

Marchi, B. de, Funtowicz, S., Lo Cascio, S., Munda, G., 2000 b. Combining Participative and Institutional Approaches with Multicriteria Evaluation. An Empirical Study for Water Issues in Troina, Sicily, Ecological Economics, 34, 267-282.

Mitchell, R.C., Carson R., 1989. Using Surveys to Value Public Goods: the Contingent Valuation Methods, Washington, D.C., Resources for the Future.

Mitchell, R.K., Agle, B.R., Wood, D.J., 1997. Towards a Theory of stakeholder Identification and Salience: Defining the principle of Who and What Really Counts, Academy of Management Review, 22, 4, 853-86.

O'Connor, M., Van den Hove, S., 2001. Prospects for Concertation on Nuclear Risks and Technological Options: Innovations in Governance Practices for Sustainable
Development in the European Union, Journal of Hazardous Materials, 86, 77-99.

O'Connor, M., Meite, V. (Eds), 2004 forthcoming. Environmental Evaluation, Cheltenham, Edward Elgar.

Renn, O., Webber, T., Rakel, H., Daniel, P., Johnson, B., 1993. Public Participation in Decision-Making: a Three-step Procedure, Policy Sciences, 26, 3, 189-214.

Stewart, D., Shamdasani, P., 1990. Focus Groups. Theory and Practice, Newbury Park, Sage Publications.

Van den Hove, S., 2001. Approches participatives pour la gouvernance en matière de développement durable : une analyse en termes d'effets, in Froger, G. (Ed.), Gouvernance et développement durable, Bâle/Genève/Munich, Helbing \& Lichtenhahn, 53-89.

WBCSD (World Business Council for Sustainable Development), 1999. Meeting Changing Expectations: Corporate Social Responsibility, progress report, March 1999, http://www.wbcsd.org.

Reçu le 23 mai 2003. Accepté le 4 décembre 2003.

To access this journal online: www.edpsciences.org 